\title{
Clinical Characteristics Affecting Lobeglitazone Response in Patients with Type 2 Diabetes
}

Bong Soo Cha' ${ }^{1}$, Ji-Yeon Lee ${ }^{1}$, Yongin Cho', Minyoung Lee ${ }^{1}$, Yong-ho Lee ${ }^{1}$, Byung-Wan Lee', Eun Seok Kang1

${ }^{1}$ Department of Internal Medicine, Yonsei University College of Medicine, Seoul, Korea

\section{Background}

Lobeglitazone (Duvie) is a novel thiazolidinedione, currently used for the treatment of type 2 diabetes (T2DM) in Korea. We investigated clinical predictive parameters responsible for the glycemic response of lobeglitazone in patients with T2DM.

\section{Subjects and Methods}

A total of 423 patients with T2DM who were treated with lobeglitazone for more than 6 months were analyzed retrospectively. Good responders were defined when patients experience a decrease of hemoglobin $\mathrm{A} 1 \mathrm{c}(\triangle \mathrm{HbA} 1 \mathrm{c})$ of $\geq 1 \%$. Multivariate logistic analyses were performed to identify parameters predictive of lobeglitazone treatment.

\section{Results}

Table 1. Phenotype comparison between patients according to lobeglitazone response

\begin{tabular}{llll}
\hline Variables & $\begin{array}{l}\text { Poor } \\
\text { responder } \\
(\mathbf{n}=\mathbf{1 1 5})\end{array}$ & $\begin{array}{l}\text { Good } \\
\text { responder } \\
(\mathbf{n}=\mathbf{1 2 0})\end{array}$ & $\mathbf{P}$ \\
\hline Age, years & $62.4 \pm 12.0$ & $63.8 \pm 12.7$ & 0.304 \\
Male sex & $93(62.4)$ & $95(55.6)$ & 0.214 \\
DM duration, years & $6.0 \pm 3.7$ & $5.8 \pm 3.8$ & 0.657 \\
Medication duration, days & $347.6 \pm 114.0$ & $349.9 \pm 116.9$ & 0.857 \\
Body mass index, kg/m² & $24.0 \pm 3.4$ & $24.7 \pm 4.1$ & 0.086 \\
HbA1c, \% & $7.2 \pm 1.4$ & $8.7 \pm 1.3$ & $<0.001$ \\
FPG, mg/dL & $143.2 \pm 52.7$ & $172.6 \pm 57.9$ & $<0.001$ \\
PP2, mg/dL & $224.4 \pm 92.7$ & $280.5 \pm 84.3$ & $<0.001$ \\
Fasting C-peptide, ng/mL & $2.4 \pm 1.8$ & $3.2 \pm 2.2$ & 0.007 \\
Fasting insulin, $\mu \mathrm{U} / \mathrm{mL}$ & $8.9 \pm 8.3$ & $12.0 \pm 10.6$ & 0.023 \\
HOMA-IR & $3.6 \pm 5.7$ & $5.1 \pm 5.5$ & 0.056 \\
HOMA-B & $45.1 \pm 37.7$ & $59.2 \pm 77.1$ & 0.104 \\
Total cholesterol, mg/dL & $158.1 \pm 40.2$ & $165.5 \pm 38.8$ & 0.097 \\
Triglyceride, mg/dL & $130.6 \pm 101.9$ & $150.4 \pm 87.2$ & 0.066 \\
HDL-C, mg/dL & $46.7 \pm 12.1$ & $44.4 \pm 11.4$ & 0.086 \\
LDL-C, mg/dL & $85.5 \pm 30.9$ & $92.0 \pm 30.4$ & 0.185 \\
BUN, mg/dL & $18.0 \pm 8.4$ & $19.8 \pm 9.6$ & 0.078 \\
Creatinine, mg/dL & $1.1 \pm 0.9$ & $1.0 \pm 0.6$ & 0.644 \\
AST, IU/L & $23.4 \pm 13.0$ & $25.7 \pm 26.3$ & 0.348 \\
ALT, IU/L & $24.7 \pm 18.7$ & $29.2 \pm 36.8$ & 0.182 \\
Other antidiabetic drugs & & & \\
Insulin & $34(22.8)$ & $51(29.8)$ & 0.157 \\
Metformin & $88(59.1)$ & $101(59.1)$ & 0.999 \\
Sulfonylurea & $39(26.2)$ & $39(22.8)$ & 0.484 \\
DPP4 inhibitor & $45(30.2)$ & $69(40.4)$ & 0.059 \\
\hline & & & \\
\hline
\end{tabular}

Table 2. Changes in anthropometric and biochemical parameters

\begin{tabular}{llll}
\hline Variables & Baseline & Follow up & P-value \\
\hline HbA1c, \% & $7.5 \pm 1.1$ & $6.9 \pm 1.0$ & $<0.001$ \\
FPG, mg/dL & $146.4 \pm 40.7$ & $132.5 \pm 35.6$ & $<0.001$ \\
PP2, mg/dL & $230.5 \pm 73.2$ & $207.2 \pm 67.3$ & $<0.001$ \\
Fasting C-peptide & $2.7 \pm 1.8$ & $2.0 \pm 0.9$ & $<0.001$ \\
Fasting insulin & $10.8 \pm 12.1$ & $8.0 \pm 5.0$ & $<0.001$ \\
HOMA-IR & $4.2 \pm 5.5$ & $2.6 \pm 1.8$ & $<0.001$ \\
HOMA-B & $54.8 \pm 70.7$ & $49.2 \pm 35.9$ & 0.582 \\
Total cholesterol, mg/dL & $161.6 \pm 37.6$ & $165.9 \pm 35.4$ & 0.020 \\
Triglyceride, mg/dL & $146.5 \pm 86.3$ & $133.4 \pm 81.3$ & 0.003 \\
HDL-C, mg/dL & $44.7 \pm 11.0$ & $48.6 \pm 12.2$ & $<0.001$ \\
LDL-C, mg/dL & $88.6 \pm 31.9$ & $89.8 \pm 29.3$ & 0.371 \\
BUN, mg/dL & $17.9 \pm 8.6$ & $18.2 \pm 7.8$ & 0.353 \\
Creatinine, mg/dL & $1.0 \pm 0.7$ & $1.0 \pm 0.7$ & 0.787 \\
AST, IU/L & $25.5 \pm 19.5$ & $23.8 \pm 14.1$ & 0.071 \\
ALT, IU/L & $28.9 \pm 28.4$ & $23.9 \pm 17.9$ & $<0.001$ \\
Body weight, kg & $66.2 \pm 12.2$ & $67.4 \pm 12.2$ & $<0.001$ \\
Body mass index, $\mathrm{kg} / \mathrm{m}^{2}$ & $24.8 \pm 3.6$ & $25.3 \pm 3.7$ & $<0.001$ \\
\hline & & &
\end{tabular}

Table 3. Multiple regression analysis for better glycemic response of lobeglitazone

\begin{tabular}{|c|c|c|c|c|}
\hline \multirow[b]{2}{*}{ Variables } & \multicolumn{2}{|c|}{ Model 1} & \multicolumn{2}{|c|}{ Model 2} \\
\hline & $\beta$ & $\mathrm{P}$ & $\beta$ & $P$ \\
\hline Age, years & 0.002 & 0.571 & -0.001 & 0.804 \\
\hline Sex, female & 0.172 & 0.103 & 0.077 & 0.506 \\
\hline Initial BMI, kg/m² & 0.040 & 0.005 & 0.032 & 0.038 \\
\hline DM duration, years & -0.021 & 0.148 & -0.055 & $<0.001$ \\
\hline Baseline $\mathrm{HbA} 1 \mathrm{c}, \%$ & 0.563 & $<0.001$ & 0.553 & $<0.001$ \\
\hline Fasting C-peptide, $\mathrm{ng} / \mathrm{mL}$ & 0.088 & 0.019 & 0.066 & 0.042 \\
\hline Triglyceride, $\mathrm{mg} / \mathrm{dL}$ & 0.001 & 0.369 & 0.001 & 0.535 \\
\hline
\end{tabular}

Model 1: unadjusted

Model 2: age, sex, initial BMI, diabetes duration, baseline $\mathrm{HbA1c}$, fasting C-peptide level, and triglyceride adjustment.

\section{Conclusion}

After lobeglitazone administration for median 350 days, mean $\mathrm{HbA} 1 \mathrm{c}$ levels were decreased by $0.6 \%$ from $7.5 \pm 1.1 \%$ to $6.9 \pm$ $1.0 \%$. In the multivariate analysis, higher $\mathrm{BMI}$, shorter diabetes duration, higher baseline $\mathrm{HbA} 1 \mathrm{c}$ and fasting C-peptide level remained independent predictors for a good response (all $P<0.05$ ). Regarding adverse effects, weight gain was $1.3 \mathrm{~kg}$, from $65.6 \pm$ $12.1 \mathrm{~kg}$ to $66.9 \pm 12.5 \mathrm{~kg}$, and there was no serious side effects.

With lobeglitazone treatment, a better response can be expected in obese T2DM patients with inadequate glycemic control, longstanding diabetes, and severe insulin resistance. 